\title{
A Brief Survey on Modern Iris Feature Extraction Methods
}

\author{
Hanaa M. Ahmed a, Mohammed A. Taha ${ }^{b^{*}}$ \\ ${ }^{a}$ University of Technology, Baghdad, Iraq.110113@uotechnlogy.edu.iq \\ ${ }^{\text {b } U n i v e r s i t y ~ o f ~ T e c h n o l o g y, ~ B a g h d a d, ~ I r a q . ~ c s .19 .54 @ g r a d . u o t e c h n o l o g y . e d u . i q ~}$ \\ *Corresponding author.
}

Submitted: 15/04/2020

Accepted: 11/06/2020

Published: 25/01/2021

\section{K E Y W O R D S}

Biometric System,

Iris Feature Extraction,

Iris recognition,

Texture analysis.

\section{A B S T R A C T}

This paper presents a survey of some of the most recent research work on the extraction phase of the Iris recognition system. Iris biometric system has been used for person recognition in wide applications where person identity authentication required for its stability and uniqueness as compared to other biometric systems. Iris based recognition systems consist of stages are Iris localization, normalization, feature extraction, and matching. The extraction of features influences the precision and reliability of the biometric system, so several methods have been used.

\footnotetext{
How to cite this article: H. M. Ahmed, and M. A. Taha, "A Brief Survey on Modern Iris Feature Extraction Methods, "Engineering and Technology Journal, Vol. 39, Part A, No. 01, pp. 123-129, 2021.

DOI: https://doi.org/10.30684/etj.v39i1A.1680

This is an open access article under the CC BY 4.0 license http://creativecommons.org/licenses/by/4.0
}

\section{INTRODUCTION}

Personal identification is one of the most essential features of the security system. In recent years, biometric systems have become increasingly relevant to the functioning of the security system. Biometric refers to the measurable investigation of natural characteristics that are used in numerous technologies for individual identification, such as e.commerce, military, communication, etc. Biometric takes advantage of physiological or behavioral properties. Biometric, this quantifiable feature can be physical, such as the face, iris, distinctive fingerprint, hand geometry, and speech or behavioral, such as signature and beat writing [1]. Because of its uniqueness and stability over time, Iris is one of the most important biometric which is used for security concerns. It is a color circular piece of the human eye. The main function of the human iris is to manage the amount of lighting reaching the retina by controlling the diameter and size of the pupil. The iris is one of the inner parts of the human body, and it is secured by the cornea and eyelid [2]. Iris based recognition system is one of the most reliable security systems in recent years [3]. Iris has an exceptional sum of special points of interest, as outlined in Fig1. There are many ways to retrieve iris features since each method gives a specific recognition rate; therefore, this work aims to review and compare modern methods through recognition rate in biometric systems. 
The rest of this paper is organized as follows. Section 2 gives the main steps of iris-based recognition systems. Sections 3 and its subsections list briefly explain the most common methods used for iris texture feature extractions, followed by sections 4, which its Results and discussion, and section 5 are conclusions.

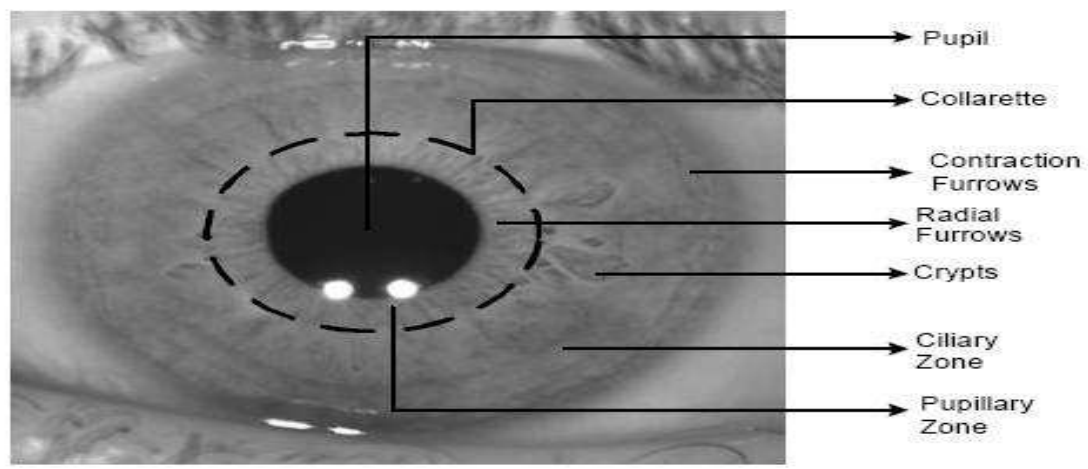

Figure 1: Iris images [4]

\section{2- IRIS RECOGNITION SYSTEM}

The essential stages of an iris recognition system are [5]:

\section{Image acquisition:}

The first stage consists of acquiring an input image from various devices. The quality of the image affects the overall performance of the recognition system.

\section{Iris localization:}

This stage aims to localize the circular zone between the pupil and the sclera region to extract its features.

\section{Normalization:}

The resolution of the sensor, which is used to capture an image and the imaging distance affects the estimate of the iris due to understudy expansion and withdrawal [6]. To address such varieties in the measure, the sectioned iris is more often than not unwrapped and transformed into a normalized arrange system. Normalization is the process of representing the sectioned circular iris as a rectangular form[7]. A broadly well-known procedure for iris normalization, Daugman's rubber sheet model, convert all points within the segmented iris to a match of polar arranges [8].

\section{Feature extraction stage and matching:}

The most important stage of a recognition system is feature extraction, which is responsible for extracting and encode the main feature and key points from the normalized iris region as the feature vector. Feature vector will be used to compare the test image with the other feature vectors stored in the database in the matching stage [6]. Fig 2 summarizes the overall stages of the recognition system. 


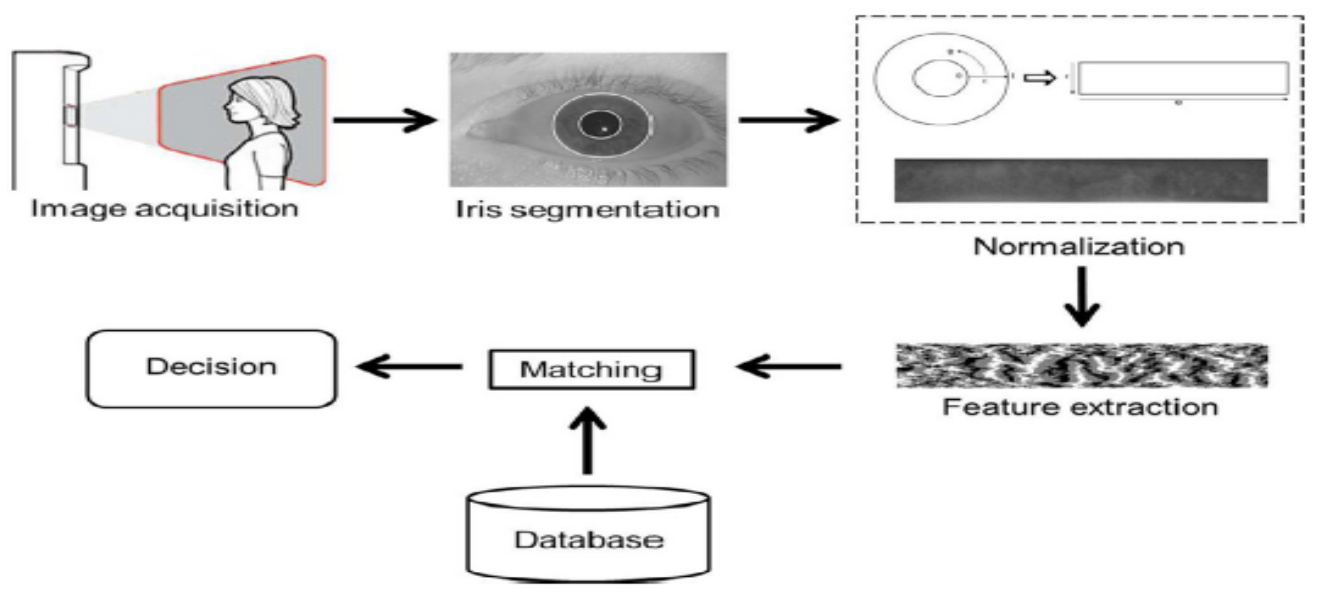

Figure 2: Iris recognition system [5].

\section{3- Iris Features EXtraction Methods}

The iris region has a unique surface; in this way, each person can be precisely identified. The feature extraction strategies return the display of specific information in the iris area. It evaluates a few critical characteristics in an iris. Feature extraction methods can be divided into two groups [9] ;

1) Image or signal processing techniques consist of frequency and spatial domain.

2) Statistical processing techniques that analyze the spatial distribution of gray values.

Some of these methods are investigated in this work.

\section{A. Gabor filter}

The first method was used for iris recognition systems introduced by Daugman [6 - 10 -11]. This method involves surface division includes subdividing a picture into differently textured segments based on a filter-bank demonstration called Gabor filter. Gabor basic capacities are modulated sinusoidal functions by Gaussian functions. The features of iris are obtained by apply two-dimension (2D) Gabor filters. A few banks of Gabor filters are applied on normalized iris image, including 15, 20, 25, 30, 35 filters by shifting filters orientation and wavelengths. Two-dimension Gabor filter can be shown as Eq (1) [12]

$$
\begin{aligned}
\mathrm{G}(\mathrm{x}, \mathrm{y}, \theta, \mathrm{f})= & e\left\{-\frac{1}{2}\left[\frac{\mathrm{x} / 2}{\partial x^{\prime 2}}+\frac{y^{\prime 2}}{\partial \mathrm{y}^{\prime 2}}\right]\right\} * \cos \left(2 \pi f x^{\prime}\right) \\
\mathrm{x}^{\prime} & =x \cos \theta+y \sin \theta \\
\mathrm{y}^{\prime} & =y \cos \theta-x \sin \theta
\end{aligned}
$$

where:

$\partial \mathrm{x}$ : filters spatial size.

$\theta$ : angle orientation.

f: frequency of the filter.

When an iris image is convolved with a bank of 20 filters, the Gabor filter has twenty imaginary and twenty real parts. A 2024 bit size feature vector, which is invariant to contrast the position of camera and illumination, was obtained by combing both imaginary and real parts [13].

\section{B. Log-Gabor Filter}

Log Gabor filter was used by P. Yaoand in [8], which is a signal processing technique similar to the Gabor filter presented by John Daugman [6]. It was used in a wide application in computer vision such as iris and fingerprint extraction. In common, whereas adjusted Log-Gabor is used to return the iris features rather than complex Gabor filter utilized in Daugman's strategy. The main benefit of Log- Gabor filter over Gabor filter is the previous are entirely bandpass channels and the last mentioned is not. This property makes the Log-Gabor filters more reasonable to return the iris region's feature in any case of the foundation brightness. log-Gabor filters are used Gaussian transfer function on the logarithmic scale. Iris pictures are better coded by the log Gabor filter that has 
Gaussian function when seen on the logarithmic scale. The recurrence reaction of a log-Gabor can be given as $\mathrm{Eq}(4)[9]$ :

$$
\mathrm{G}(\mathrm{f})=\exp \left\{-0.5 \times \log (f / f O)^{2} / \log (\sigma / f O)^{2}\right\}
$$

where

f0: center frequency

$\sigma:$ bandwidth of the filter

Log-Gabor minimizes the feature vector, but computational complexity was increased [2].

\section{Discrete Cosine Transform (DCT)}

Discrete cosine transform (DCT) is a signal processing technique that was used to extract iris features with low computation complexity and high accuracy as used in [14]. Input data is represented as a sum of sinusoids of varying magnitudes and frequencies in DCT. DCT is similar to Fourier transform (FT) in used sinusoidal premise capacities, but it differs between them is that cosine transform premise capacities are not complex; they utilize as it were only the real part (cosine function) and not sine function as a Fourier transform [15]. Signal was decomposed into its basic components when DCT was used. It compresses all the information of the iris and concentrates it in a few coefficients found within the upper-left corner.2-D discrete cosine transform (DCT) condition for an $\mathrm{N} \times \mathrm{N}$ picture is given by $\mathrm{Eq}(5)[16]$ :

$$
F(u, v)=c(u) c(v) \sum_{x=1}^{n-1} \sum_{x=1}^{n-1} f(x, y) \cos \frac{(2 x+1) u \pi}{2 n} \cos \frac{(2 x+1) v \pi}{2 n}
$$

where

$$
\begin{aligned}
& C(u)=C(v)=\frac{1}{\sqrt{2}} \text { for } u, v=0 \\
& C(u)=C(v)=\sqrt{\frac{2}{n}}, \text { for } u, v \neq 0
\end{aligned}
$$

C $(u, v)$ is called DCT coefficients, and I(r,c) is the iris image data. Since this change employments, as it were the cosine work, it can be calculated utilizing on real number (not complex). The imperative include of the DCT, the include produces it so valuable in information compression, is that it takes related input information and concentrates its vitality in fair the primary few change coefficients [16].

\section{Discrete Wavelet Transform (DWT)}

As seen in [17], 2D DWT has been used in the iris feature extraction to reduce processing time and obtain a minimum reduction size. Wavelets' primary vital application is to prepare, transform data, and particularly compress image data. Compared to the DCT, DWT's essential benefit is that the DWT is located in both spatial and recurrent spaces [18]. In this way, a given signal shift will contain both spatial and recurrence data from that signal. The Wavelet Transform process is a simple concept. The original transformed of the iris was divided into 4 new sub-iris called LL, LH, HL, and $\mathrm{HH}$ based on its frequency and spatial components to replace it. Each sub-iris is $1 / 4$ times the original iris as shown in fig 3 [19].
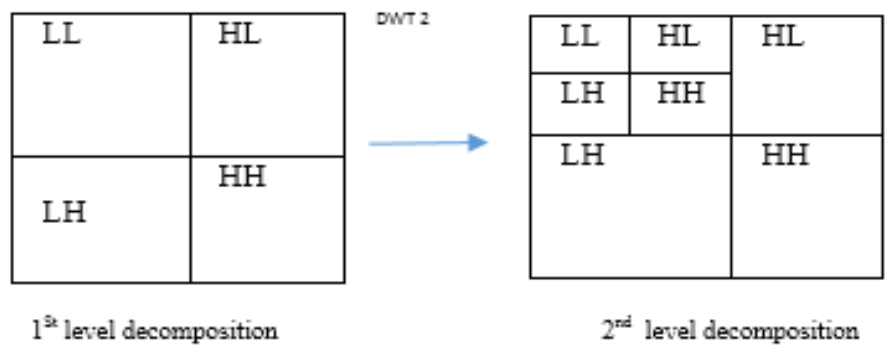

Figure 3: Wavelet decomposition level 


\section{E. Principal Component Analysis (PCA)}

The principal component analysis has been used in [20-21-22] to extract features from the iris. PCA is a statistical technique that was used as a dimension reduction in various applications such as image compression, data analysis, data visualization, feature extraction, and pattern recognition. By using mathematical methods, it is used to transform large numbers of correlated variables into principal components, which are smaller uncorrelated variables. The primary principle component accounts for as much of the changeability within the information as conceivable, and each succeeding component accounts for as much of the remaining changeability as conceivable. The principal Component methodology permits the measurement of the eigenvalue deterioration of an information covariance matrix or specific regard weakening of an information matrix, more regularly than not after mean focusing the data for each quality [23]. Spatial global features are usually presented by the PCA.

\section{F. Gray Level Co-occurrence Matrix (GLCM)}

GLCM is a second-order statistic that estimates the properties of two or more-pixel values occurring in particular areas almost equivalent to one another. GLCM may be widely used as an iris texture examination strategy. It improves the points of interest for the image and gives a clarification. It improves the points of interest in the image and clarifies it. The GLCM could be a tabulation form of how often a diverse collection of grayscales takes place in an iris image [21]. In GLCM, the number of rows and columns is precisely increasing to the number of pixel brightness values within the image occurrence matrices, which develop completely different distances and orientations. The distance determines offset between reference and neighbor cells; increasing the distance increases the scale of the texture being sampled. A measurable GLCM-based extraction feature approach was used in $[24,25]$.

\section{G. Local Binary Patterns (LBP)}

In $[26,27]$ LBP was used for the extraction of iris texture features. Due to its quick performance and simple implementation, it is used for the classification of images in computer vision. By definition, for a pixel located at the point $(\mathrm{x}, \mathrm{y})$ of the local binary pattern, there is a double comparison of its value with the 8 neighbors (right-top, right-middle, right-bottom, left-top, etc.). In case the center pixel's esteem is more noteworthy than the neighbor's esteem, type "1." Something else, type " 0 " in the center of the $3 * 3$ mask. This gives an 8-digit binary number (which is usually converted to a decimal number for convenience).LBP is working with a gray level image, so colored images should be converted to a gray level [28].

\section{H. Sift descriptor (Scale-invariant Feature Transform)}

Scale-invariant Feature Transform (SIFT) has been used for iris extraction features by D. G. Lowe [29 It was used for extraction of features and pattern recognition because of its robustness to scale, rotation, and change in illumination [30]. SIFT suggested an iris-based extraction system with few sub-regions alone. In this procedure, from cleared out of the iris, sub locales are trimmed underneath the understudy, center iris, 3 sides of iris, and 2 sides of iris. Filter descriptors, when connected to these sub-districts, provide low computational complexity with high accuracy for subdistricts taken from 2 sides of the iris.

\section{4-RESULTS AND DISCUSSIONS}

Table 1 summarizes the recognition rate for various extraction methods.

TABLE I: Recognition rate comparison

\begin{tabular}{ccc}
\hline \hline No & Feature extraction methods & Recognition rate \\
\hline 1 & Gabor filter & $99.9 \%$ \\
\hline 2 & Log-Gabor Filter & $92.4 \%$ \\
\hline 3 & Discrete Cosine Transform (DCT) & $96 \%$ \\
\hline 4 & Discrete Wavelet Transform(DWT) & $99.18 \%$ \\
\hline 5 & Principal Component Analysis(PCA) & $90.2 \%$ \\
\hline 6 & Gray Level Co-occurrence Matrix(GLCM) & $99.5 \%$ \\
\hline 7 & Local Binary Patterns (LBP) & $99.87 \%$ \\
\hline 8 & Scale-invariant Feature Transform (SIFT) & 97.4 \\
\hline
\end{tabular}


As seen in Table I, a high recognition rate was obtained from the signal processing methods such as the Gabor filter, but with high computation cost, which leads to increase enrolment and verification time of the recognition system. Log Gabor filter minimizes the size of the feature vector, but less recognition rate as compared with the Gabor filter. DWT has small size feature vector, but some frequency component of the iris has been lost. DCT gives the smallest size of the feature vector as compared with other methods based on the strategies reviewed; statistical methods such as GLCM and LBP have been shown to provide low computational complexity and a small-scale feature vector leading to an increase in the speed of the recognition system. The SIFT procedure has the benefit of eliminating the iris normalization phase, which mainly reduces the complexity and computational cost of the recognition systems.

\section{5-CONCLUSION}

In recent years, Iris recognition systems are widely used in different applications. Based on the field of application, the different strategies utilized for iris feature extraction. Some of these strategies are surveyed in this paper. Based on the strategies reviewed, it is observed statistical methods such as GLCM and LBP have been shown to provide low computational cost and a high recognition rate, leading to an increase in the speed of the recognition system. This works can provide a platform for new feature extraction methods in iris-based recognition systems as future works.

\section{References}

[1] A. G. Gale1, DR. S. S. Salankar, A Review On Advance Methods Of Feature Extraction In Iris Recognition System, IOSR Journal of Electrical and Electronics Engineering (IOSR-JEEE) e-ISSN: 22781676, p-ISSN: 2320-3331 PP 65-70, International Conference on Advances in Engineering Technology 2014 (ICAET-2014).

[2] M. H. Hamd, Optimized biometric system based iris-signature for human identification, Int. J. Adv. Intell. Informatics., 5(2019) 273-284. https://doi.org/10.26555/ijain.v5i3.407

[3] A. Azizi, H. R. Pourreza, Efficient IRIS Recognition Through Improvement of Feature Extraction and Subset Selection, Int. j. comput. sci. inf. technol. secur., 2(2009). https://doi.org/10.48550/arXiv.0906.4789

[4] C. Boyce, A. Ross, M. Monaco, L. Hornak, and X. Li., 2006. Multispectral iris analysis: a preliminary study, In the Proceedings of Computer Vision and Pattern Recognition Workshop (CVPRW'06), pp: 51. https://doi.org/10.1109/CVPRW.2006.141

[5] R. R. Jillelaa, A. Ross, Segmenting iris images in the visible spectrum with applications in mobile biometrics, Pattern. Recognit. Lett., 31 October 2014. http://dx.doi.org/10.1016/j.patrec.2014.09.014

[6] J. Daugman, High Confidence Visual Recognition of Persons by a Test of Statistical Independence, IEEE Trans. Pattern Anal., (1993).

[7] K. W. Bowyer, K. Hollingsworth, P. J. Flynn, Image understanding for iris biometrics: A survey, Comput. Vis. Image Underst., 110 (2008) 281-307. https://doi.org/10.1016/j.cviu.2007.08.005

[8] P. Yao, J. Li, X. Ye, Z. Zhang, and B. Li. Iris Recognition Algorithm Using Modified Log-Gabor Filters, Proc. Conf. Pattern. Recognit., (2006). http://dx.doi.org/10.1109/ICPR.2006.726

[9] Fasna KK, Athira P, Remya Krishna J S, A Review on Iris Feature Extraction Methods, Int. j. eng. res. generic. sci., 4(2016) 2091-2730.

[10] J. Daugman, How Iris Recognition works, proceedings of 2002 International Conference on Image processing, 1 (2002). https://doi.org/10.1109/ICIP.2002.1037952

[11] J. Daugman, How Iris Recognition Works, IEEE TRANSACTIONS ON CIRCUITS AND SYSTEMS FOR VIDEO TECHNOLOGY, 14( 2004).

[12] S. Minhas, M. Y. Javed, Iris Feature Extraction Using Gabor Filter, Int. Conf. Eng. Emerg. Technol., IEEE 2009. http://dx.doi.org/10.1109/ICET.2009.5353166

[13] S. Minhas, M. Y. Javed, Iris Feature Extraction Using Gabor Filter, Int. Conf. Eng. Emerg. Technol., 2009, 978-1-4244-5632-1/09, IEEE. http://dx.doi.org/10.1109/ICET.2009.5353166

[14] A. M. Sarhan, Iris Recognition Using Discrete Cosine Transform and Artificial Neural Networks, J. Comput. Syst. Sci., 5 (2009) 369-373, 2009, ISSN 1549-3636. 
[15] A. Nichal, P. Jadhav, V. Nikam, and V. Hipparka, DCT Based Iris Feature Extraction and Recognition for Security System, Int. j. adv. res. comput., 3(2014) 2014.

[16] M. H. Abhiram, C. Sadhu, K. Manikantan, and S. Ramachandran, Novel DCT Based Feature Extraction for Enhanced Iris Recognition, 2012 International Conference on Communication, Information Computing Technology(ICCICT), Oct. 19-20, Mumbai, India. https://doi.org/10.1109/ICCICT.2012.6398164

[17] K. M. Ali Alheeti, Biometric Iris Recognition Based on Hybrid Technique, Int. J. Soft. Comput., 2( 2011)1-9. http://dx.doi.org/10.5121/ijsc.2011.2401

[18] W. Boles and B. Boashash. A human identification technique using images of the iris and wavelet transform, IEEE. Trans. Signal. Process., 46 (1998). http://dx.doi.org/10.1109/78.668573

[19] D. Gupta, S. Choubey, Discrete Wavelet Transform for Image Processing, Int. J. Emerging Technol. Adv. Eng., 4(2015).

[20] J. Xin Sh, X.Feng Gu, The Comparison of Iris Recognition Using Principal Component Analysis, Independent Component Analysis and Gabor Wavelets, ICCSIT, 2010, IEEE.

[21] R., M., Haralick, K., Shanmugam, and I. Dinstein Textural Features for Image Classification, IEEE Trans. on Systems, Man and Cybernetics (1973)610 - 621. https://doi.org/10.1109/TSMC.1973.4309314

[22] P. S. R. Chandra Murty, and E. S. Reddy, Iris recognition system using Principal Components of texture characteristics. TECHNIA-Int. J. Computing Science and Communication Technologies 2( 2009)343-348.

[23] Y. A. Jasim, A. A. Al-Ani, and L. A. Al-Ani, Iris Recognition Using Principal Component Analysis, nt. Conf. Inf. Commun. Syst., IEEE 2018. http://dx.doi.org/10.1109/AiCIS.2018.00028

[24] R. A. Rasool, Iris Feature Extraction and Recognition based on Gray Level Co-occurrence Matrix (GLCM) Technique, Int. J. Comput. Appl., 181 ( 2018) 0975 - 8887.

[25] K. Hajari, U. Gawande, and Y. Golhar, Neural Network Approach to Iris Recognition in Noisy Environment, Int. J. Inf. Secur. Priv., (ICISP2015), 11-12 December 2015, Nagpur, INDIA, Available online at

[26] P. Rasti, M. Daneshmand, and G. Anbarjafari, Statistical approach based iris recognition using local binary pattern, Cod. 7997 | Tecnología de la construcción | 3305.05 Tecnología del hormigón ,2017 (2017) . http://dx.doi.org/10.6036/7997

[27] D. G. Lowe, Distinctive Image Features from Scale-Invariant Keypoints , Int. J. Comput. Vis., (2004).

[28] M. Z. Rashad, M. Y. Shams, O. Nomir, and R. M. El-Awady, IRIS RECOGNITION BASED ON LBP AND COMBINED LVQ CLASSIFIER , Int. j. comput. sci. inf. technol., 3( 2011). http://dx.doi.org/10.5121/ijcsit.2011.3506

[29] D. G. Lowe, Distinctive Image Features from Scale-Invariant Keypoints , Int. J. Comput. Vis., 2004.

[30] M. Ibrahim, A. Eleyan, and B. Karlik. Sift-based iris recognition using sub-segments. International Conference on Technological Advances in Electrical, Comput. Electr. Eng., (2013)350- 353. 